\title{
ETUDE DES MÉTHODES DE DÉTERMINATION DES PERFORMANCES LAITIÈRES
}

\section{II. - LA PRÉCISION D'UN CONTROLE LAITIER A PERIODICITE VARIABLE}

PAR

CARRÉ (D.), POLY (J.), VISSAC (B.) (1)

Laboratoire de recherches de Zootechnie, Institut national Agronomique, Paris.

\section{PLAN DU MÉMOIRE}

I. - Les sources d'erreur du contrôle laitier.

I,I. - Erreurs physiques.

I,2. - Erreurs humaines.

I,3. - Incertitude statistique liée à l'échantillonnage périodique de la lactation.

II. - Les travaux antérieurs.

III. - Matériel et méthodes utilisés.

IV. - Première étude : données de la Pierre-qui-Vire.

V. - Deuxième étude : données du syndicat de la Manche.

\section{Conclusions.}

\section{I. - LES SOURCES D'ERREURS DU CONTROLE LAITIER}

La pratique du contrôle laitier entraîne plusieurs types d'erreurs :

I,I. - Des erreurs physiques, liées aux instruments et aux méthodes de mesures ;

I,2. - Des erreurs humaines, attachées à la qualité du travail réalisé par l'agent de contrôle ;

I,3. - Enfin, une erreur statistique résultant d'un échantillonnage périodique de la lactation.

Nous étudierons presque exclusivement dans le présent mémoire les problèmes soulevés par ce dernier chapitre.

(1) Avec la collaboration technique de Deotre (J. C.) et l'aide matérielle du Herd-Book Normand. qui a gracieusement mis à notre disposition son installation mécanographique IBYI. 


\section{1,1. - Erreurs physiques.}

Il n'est pas dans notre intention d'approfondir ce paragraphe, car nous ne connaissons pas d'études valables sur cette question. Nous nous proposons seulement d'en discuter certains aspects théoriques, faute d'une expérimentation en la matière.

\section{1, 1. 1. Causes des erreurs physiques.}

(I) Précision de la pesée de lait.

Une balance romaine fournit dans les meilleures conditions une pesée à Ioo grammes près; les accords européens (I2) qui admettent une pesée à $200 \mathrm{~g}$ près sont d'ailleurs beaucoup plus tolérants. La quantité de lait produite en I jour par une vache est déterminée par 2 ou 3 pesées, dans la mesure où la production correspondant à une seule traite ne nécessite qu'une pesée.

(2) Précision des prélèvements dits proportionnels.

Le contrôleur prélève, à l'aide d'une pipette graduée au $\mathrm{I} / \mathrm{IO}$ de $\mathrm{cm}^{3}$, un volume de lait proportionnel à la quantité de lait recueillie à chaque traite, pour un animal donné. Il constitue ainsi un échantillon composite de $40 \mathrm{~cm}^{3}$ environ, où le taux butyreux de chaque traite est pondéré par la production laitière correspondante.

L'erreur physique sur le taux butyreux qui découle d'erreurs de lecture dans la pipette, pour réaliser l'échantillon proportionnel, est absolument négligeable comme le montre l'exemple ci-après.

Une vache produit $20 \mathrm{~kg}$ de lait.

Traite du soir : $8 \mathrm{~kg}$ à $T B_{1}$-prélèvement de $\mathbf{I} 6 \mathrm{cc} \pm 0, \mathbf{I} \mathrm{cc}$;

Traite du matin : I2 $\mathrm{kg}$ à $\mathrm{TB}_{2}$-prélèvement de $24 \mathrm{cc} \pm \mathrm{O}, \mathrm{I} \mathrm{cc}$.

La quantité totale de matière grasse $\mathrm{MG}_{t}$ contenue dans le prélèvement proportionnel $\mathrm{MG}$ peut s'exprimer :

$$
\begin{aligned}
\mathrm{MG}_{t} & =\mathrm{TB}_{1}(\mathrm{I} 6 \pm 0, \mathrm{I})+\mathrm{TB}_{2}(24 \pm 0, \mathrm{I}) \\
\mathrm{MG}_{t} & =\mathrm{TB}_{1} \cdot \mathrm{I} 6\left(\mathrm{I} \pm \frac{0, \mathrm{I}}{\mathrm{I} 6}\right)+\mathrm{TB}_{2} \cdot 24\left(\mathrm{I} \pm \frac{\mathrm{O}, \mathrm{I}}{24}\right) \\
& =\mathrm{MG} \pm \frac{\mathrm{TB}_{\mathrm{i}}}{\mathrm{IO}} \pm \frac{\mathrm{TB}_{2}}{\mathrm{IO}} \\
& =\mathrm{MG}\left(\mathrm{I} \pm \frac{\mathrm{TB}_{1}}{\mathrm{I} 0 \cdot 40 \overline{\mathrm{TB}}} \pm \frac{\mathrm{TB}_{2}}{\mathrm{I} \cdot 40 \overline{\mathrm{TB}}}\right)
\end{aligned}
$$

Si on peut considérer que $\mathrm{TB}_{1}, \mathrm{~TB}_{2} \div \overline{\mathrm{TB}}$; l'erreur relative maximum sur la matière grasse totale contenue dans le prélèvement est donc de $\frac{I}{200}$, et sur les II cc prélevés lors du Gerber, nous aurons sensiblement une erreur absolue maximum de $\mathrm{r} / 20$ de g ce qui est absolument négligeable. 
(3) Précision sur le volume du prélèvement effectué dans l'échantillon proportionnel.

On prélève dans un flacon où a été réalisé l'échantillon composite, II $\mathrm{cm}^{3}$ de lait arec une pipette à un trait, ce qui entraîne une erreur volumétrique de 1'ordre de I p. roo qui se répercute sur le taux butyreux, mais qui est faible étant au plus égale à I p. Ioo.

\section{(4) Précision de la méthode Gerber.}

Cette méthode fournit des erreurs d'analyse très faibles ; des erreurs systématiques peuvent se produire cependant quand on est en présence de laits particulièrement riches (8), comme c'est le cas par exemple pour les laits de brebis.

En fait, la plus grande part des erreurs physiques imputables à la méthode Gerber provient de l'imprécision de la lecture sur l'échelle graduée du butyromètre où 1'on peut apprécier correctement la demi-unité soit $0,5 \mathrm{~g}$ de matière grasse au kilogramme.

Au total, nous pensons approcher d'assez près la réalité en estimant que dans le cas d'un travail correctement effectué, l'erreur absolue dans la détermination du taux butyreux est de l'ordre de I g. Fille résulte surtout d'une erreur volumétrique dans le prélèvement.

\section{1, 1. 2. Importance des erreurs physiques.}

Nous pouvons essayer de chiffrer maintenant les erreurs absolues et relatives sur les résultats d'un contrôle.

Pour la production du lait.

L'erreur absolue sur la quantité de lait, dans les meilleures conditions, pour une vache traite deux fois lors du contrôle est de l'ordre de $200 \mathrm{~g}$.

L'erreur relative correspondante est de $4 \mathrm{p}$. roo pour une production de $5 \mathrm{~kg}$, de $2 \mathrm{p}$. Ioo pour tune production de Io $\mathrm{kg}$, de $0,8 \mathrm{p}$. Ioo pour une production de $25 \mathrm{~kg}$.

Pour le taux butyreux.

Si l'erreur absolue dans la détermination du taux butyreux est de l'ordre de $\mathrm{I} g$, les erreurs relatives correspondantes pour des taux butyreux de $30 \mathrm{~g}$, $40 \mathrm{~g}$ et $50 \mathrm{~g}$ sont respectivement de $3,3 \mathrm{p}$. Ioo, 2,5 p. Ioo, 2 p. IOO.

Ponr la production de matière grasse.

I a production de matière grasse le jour du contrôle s'obtient en faisant le produit de la quantité de lait foumie par le taux butyreux de ce lait. Or, l'erreur relative sur un produit est égale à la somme des erreurs rela- 
tives sur chacun des termes qui le composent. Si l'on admet comme raisonnable une erreur relative moyenne de $3 \mathrm{p}$. roo sur le taux butyreux lors d'un contrôle, l'erreur relative dans la détermination de la quantité de lait fournie va dépendre surtout du niveau de production de l'animal le jour du contrôle, contingent lui-même des aptitudes génétiques de la vache, de sa conduite d'exploitation, de son stade de lactation. A l'échelon d'une population de résultats de contrôles, on constate en général qu'il y a une corrélation négative entre les quantités de lait fournies et la richesse en graisses de ces laits. I1 y a donc une tendance statistique à ce que les erreurs relatives sur la production de lait et sur le taux butyreux varient en sens inverse.

Quelques exemples nous donneront des bases cohérentes d'estimation de l'erreur relative sur la quantité de matière grasse lors d'un contrôle.

Exemple 1: Quantité de lait $=25 \mathrm{~kg}$. T. B. $=30-35 \mathrm{~g}$.

L'erreur relative sur la quantité de matière grasse est égale, environ, à $0,8+3,3 \sim 4$ p. Ioo.

Exemple 2: Quantité de lait $=\mathrm{I}_{5} \mathrm{~kg} . \mathrm{T} . \mathrm{B} .=40 \mathrm{~g}$.

L'erreur relative sur la quantité de matière grasse est égale, environ, à $\mathrm{I}, 3+2,5 \sim 4$ p. IOO.

Exemple 3 : Quantité de lait $=5 \mathrm{~kg}$. $\mathrm{T} . \mathrm{B} .=45 \mathrm{~g}$.

L'erreur relative sur la quantité de matière grasse est égale, environ, à $4+2,2 \sim 6$ p. IOO.

L'erreur physique relative dans la détermination de la quantité de matière grasse lors d'un contrôle, dépend principalement de la quantité de lait fournie ce jour-là. Elle varie dans la plupart des cas de 3 à 7 p. Ioo.

\section{1,2. - Erreurs humaines.}

Les erreurs humaines ne sont pas chiffrables et difficilement contrôlables ; ce sont des erreurs d'identification des animaux, des erreurs grossières de manipulations ou de mesure - certaines peuvent être systématiques, ce qui fausse évidemment considérablement les résultats enregistrés à l'échelon de toute une lactation - des erreurs enfin de transcription des données à tous les stades.

C'est, selon nous, dans la détermination des taux butyreux que les raisons d'erreurs humaines sont les plus importantes : échantillon proportionnel très mal fait, défaut d'une homogénéisation sommaire avant la prise de tout prélèvement, réalisation $d u$ "Gerber" dans de mauvaises conditions de travail. L'exécution en laboratoire des analyses de matière grasse, avec un matériel approprié, permettrait à la fois de diminuer les erreurs de contrôle et d'accélérer, par contre, la rapidité des expertises. 


\section{3. - Erreurs statistiques.}

I'incertitude statistique liée à une méthode de contróle correspond aux différences qui existent entre les productions réelles et les productions calculées à partir de contrôles plus ou moins fréquents au cours de la lactation.

Cette incertitude statistique relève de plusieurs causes : d'abord de la variabilité de la production laitière de jour à jour ; de différences plus ou moins importantes, selon les individus, dans les formes des courbes de production; de l'erreur qui est faite sur l'estimation de la durée de lactation.

Elle peut se définir par les diverses caractéristiques de la distribution de fréquence des différences entre production réelle et production calculée ; ces caractéristiques sont les suivantes :

- moyenne des valeurs arithmétiques absolues ou relatives,

- moyenne des valeurs algébriques absolues ou relatives,

- écart-type des valeurs algébriques absolues ou relatives,

- critères de normalité de la distribution.

\section{II. - LES TRAVAUX ANTÉRIEURS}

De très nombreux auteurs ont déjà étudié le problème de la précision du contrôle laitier, en fonction de la périodicité entre les contrôles. Ces auteurs ont utilisé un matériel d'étude très variable, qui était, dans de nombreux cas, formé de troupeaux expérimentaux des stations de recherches.

Les travaux anciens portent en général sur un petit nombre d'animaux, et les méthodes d'analyse statistique sont souvent insuffisantes; le paramètre le plus utilisé pour exprimer la précision du contrôle laitier est l'écart maximum enregistré entre la production contrôlée et la production réelle. Dans une étude de 1'Institut International d'Agriculture de Rome (I7) datant de I935, on peut lire en particulier que l'écart relatif maximum est le critère de précision le plus important. Mais un examen approfondi du problème nous permet de considérer que cette différence relative maximum n'a pas de valeur statistique : une forte différence maximum peut être due à un seul cas individuel. DörING (9) prouve très justement que cette différence maximum n'a aucune valeur et que le hasard peut fausser complètement les résultats lorsque l'on utilise ce paramètre.

La seule étude publiée en France se réfère à la différence relative maximum - LAPLAUd, de la Fregonière et DUFFaU (23), (24). La différence relative maximum donne des résultats extrêmement variables 


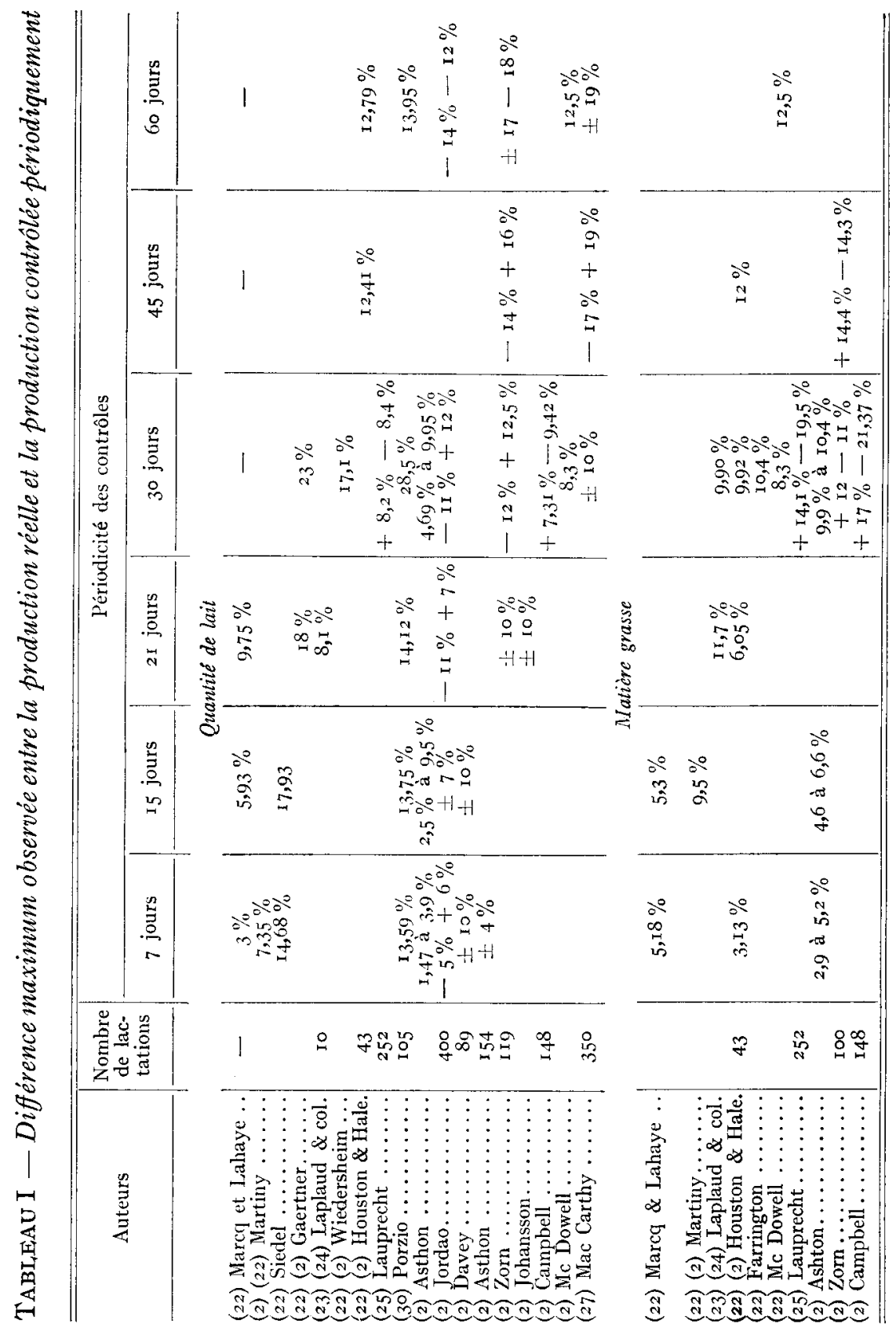


avec les auteurs et avec les pays, comme on peut le constater dans le tableau $\mathrm{I}$. Ce critère conduit même parfois à des conclusions aberrantes qui prouveraient, entre autres, que les contrôles hebdomadaire ou bimensuel ne sont pas plus précis que le contrôle bimestriel, et que ce dernier est plus précis que le contrôle mensuel (30). Toutes ces études anciennes ne présentent donc qu'un intérêt limité ; il est indispensable d'utiliser des paramètres statistiques, comme l'écart-type de la population de différences relatives entre la production réelle et la production contrôlée périodiquement pour caractériser la précision de l'échantillonnage périodique.

Un certain nombre d'études plus récentes ont utilisé les méthodes biométriques ; nous pouvons en retenir les conclusions suivantes. Selon McCARTHY et BOYLE (27) on trouve un écart-type des différences relatives entre la production réelle et la production contrôlée tous les mois, égal à $3, \mathrm{I}$ p. roo, toutes les six semaines, égal à 4,7 p. Ioo et tous les deux mois, égal à 5,4 p. Ioo ; leur étude a porté sur 350 lactations de vaches Irlandaises. En Nouvelle-Zélande, Dick (7) observe un écart-type des différences pour le contrôle mensuel de 2,39 p. Ioo. Aux Etats-Unis, nous trouvons des valeurs encore plus faibles, puisque pour le contrôle mensuel ERB et coll. (IO) trouvent un écart-type d'environ I,8 p. IOO (tableau II). Cette dernière étude est conduite sur I9 vaches Holstein de la Station expérimentale de Washington ; si l'écart-type est inférieur à celui trouvé par McCARTHY en particulier, cela est probablement dû au faible effectif du troupeau et au fait que ce troupeau d'expérience ne reflète pas les conditions réelles d'exploitation.

\section{TABLEAU II}

Valeur des écarts-types des différences entre la production contrôlée $\grave{a}$ périodicité variable et:(I) la production réelle, (2) la production contrôlée tous les mois.

\begin{tabular}{|c|c|c|c|c|c|c|c|c|c|c|}
\hline & \multicolumn{2}{|c|}{ Mensuel } & \multicolumn{2}{|c|}{ Bimestriel } & \multicolumn{2}{|c|}{ Trimestriel } & \multicolumn{2}{|c|}{4 mois } & \multicolumn{2}{|c|}{5 mois } \\
\hline & $\begin{array}{c}\text { Lait } \\
\%\end{array}$ & $\underset{\%}{\text { MI.G. }}$ & $\begin{array}{c}\text { Lait } \\
\%\end{array}$ & $\underset{\%}{\text { M. G. }}$ & $\begin{array}{c}\text { Lait } \\
\%\end{array}$ & $\underset{\%}{\text { M. G. }}$ & $\begin{array}{c}\text { Lait } \\
\%\end{array}$ & M. G. & $\begin{array}{c}\text { Lait } \\
\%\end{array}$ & $\underset{\%}{M}$ \\
\hline $\begin{array}{l}\text { (1) Ecart-type des différences rela- } \\
\text { tives par rapport à la produc- } \\
\text { tion réelle. } \\
\text { ( } 9 \text { lactations). } \\
\text { (2) Ecart-type des différences rela- } \\
\text { tives par rapport à la produc- } \\
\text { tion contrôlée mensuellement } \\
\text { (3o6 lactations). }\end{array}$ & I, 8 & 2,7 & 2,5 & 4,0 & $4, I$ & $5, \mathrm{I}$ & $4, I$ & 10,5 & 6,6 & I 1,5 \\
\hline
\end{tabular}

On peut également étudier les différences entre les productions contrôlées à des périodicités variables, le contrôle dont la périodicité est la plus forte étant utilisé comme méthode de référence. 
Le contrôle mensuel a été comparé au contrôle hebdomadaire par cette voie. La différence relative maximum trouvée par FleischmanN (I3) est alors de 22 p. Ioo et par MaRTiny, de I2 p. Ioo (22).

ERB et coll. ont comparé, comme nous l'avons fait, le contrôle mensuel à des contrôles à périodicité supérieure, en utilisant un facteur de correction saisonnier et une courbe de lactation moyenne. Les écarts types des différences relatives entre les productions contrôlées, mensuellement et tous les deux mois, 3 mois, 4 mois et 5 mois ont été calculés; nous résumons leurs résultats dans le tableau II.

Les conclusions des différents auteurs sont souvent contradictoires. La grande majorité des chercheurs européens conclut que la périodicité mensuelle est la plus longue qui puisse être envisagée, et serait plutôt favorable au contrôle tous les I5 jours et au contrôle hebdomadaire. Mais les chercheurs américains pensent que le contrôle bimestriel est amplement suffisant, comme le rapportent MARCQ et LAIIAYE (I2) qui citent alors MacDowell (28), Copeland (5), Yapp (35), GifFord (I5) et Petersen (29). Marco et Lahaye concluent d'ailleurs, en I938, que "les recherches sur la sûreté du contrôle fait tous les deux mois ne sont pas encore arrivées au point de permettre un jugement définitif ». Des auteurs américains plus récents comme ERB et coll. envisagent même, dans certains cas, le contrôle trimestriel.

Dans un ouvrage récent et très documenté, Ashron (22) termine ainsi son chapitre sur la précision du contrôle laitier : "Aucune méthode spéciale ne peut être recommandée pour toutes les organisations, tellement les conditions d'exploitation sont variées dans les différents pays. Un autre facteur de la plus grande importance est l'utilisation des résultats de contrôle laitier. Si le principal but du contrôle laitier est d'obtenir une bonne estimée de la performance d'une vache, le contrôle mensuel sera le meilleur dans la plupart des cas. Si on veut utiliser un grand nombre de performances pour tester des taureaux, le contrôle bimestriel et même le contrôle trimestriel sont admissibles. "

\section{III. - MATÉRIEL ET MÉTHODES}

Notre matériel de travail a deux origines différentes:

3,I. - Un échantillon de 5o lactations de vaches Brunes des Alpes appartenant à l'élevage de l'Abbaye de la Pierre-qui-Vire. Le contrôle volumétrique de la quantité de lait fournie au cours de chaque traite était effectué à $250 \mathrm{~cm}^{3}$ près ; le taux butyreux n'était pas connu. Devant la rareté des résultats de contrôle journalier, nous avons dû nous contenter de cet ensemble de données obtenues dans les conditions pratiques d'une ferme. Une étude plus complète portant sur des résultats de contrôles laitiers-beurriers effectués dans un autre troupeau selon 
les normes officielles (contrôle pondéral de la quantité de lait, dosage du taux butyreux par la méthode Gerber) est actuellement en cours pour vérifier les conclusions que nous avons pu établir sur cette étude préliminaire.

3,2. - Une population de 2 ooo lactations environ de vaches Normandes contrôlées tous les mois et tirées au hasard dans les archives du Syndicat de contrôle laitier de la Manche. Nous avons pu étudier sur ces données la précision du contrôle bimestriel par rapport au contrôle mensuel dans une zone assez vaste oì ce nouveau type de contrôle pourrait être appliqué à des fins de testage des taurillons.

3,3. - Les populations de différences entre la production de réfé-

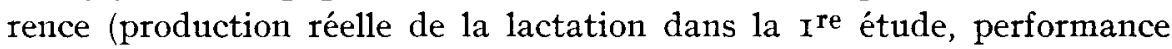
calculée à partir d'un contrôle mensuel dans $1 \mathrm{a} 2^{\mathrm{e}}$ ) et celle correspondant à chacune des diverses méthodes étudiées ont été caractérisées statistiquement par leur moyenne, leur écart-type et leurs tests de normalité : skewness et kurtosis.

\section{IV. - PREMIERE ÉTUDE. DONNÉES DE LA PIERRE-QUI-VIRE}

\section{4,1. - Résultats.}

Les performances de production laitière au cours de la lactation ont été calculées successivement :

- par la somme des productions journalières : production réelle $(\mathrm{J})$,

- à partir d'un contrôle périodique : on a supposé que des contrôles fictifs étaient réalisés tous les $21,30,45$ et 60 jours.

Le choix des dates de contrôle a été fait à l'échelon de l'étable en laissant néanmoins, comme dans la réglementation du contrôle laitier, une marge de variation aux intervalles entre contrôles successifs :

- 5 jours dans le cas d'un contrôle toutes les 3 semaines,

- 7 jours dans le cas d'un contrôle tous les mois,

- II jours dans le cas d'un contrôle tous les 45 jours,

- I3 jours dans le cas d'un contrôle tous les 2 mois.

Dans cette limite, les dates de contrôle ont été tirées au sort. Le calcul des productions a été effectué d'après le principe de la méthode Fleischmann; nous représenterons respectivement par $\mathrm{X}_{21}, \mathrm{X}_{30}, \mathrm{X}_{45}$ et $\mathrm{X}_{60}$ les performances de production laitière calculées à partir d'un contrôle tous les $2 \mathrm{I}, 30,45$ et 60 jours.

Les paramètres statistiques des populations de différences algébri- 
ques et arithmétiques entre la production réelle et celle obtenue par un échantillonnage périodique ont été calculés :

- sur les valeurs absolues d'une part (kg de lait);

$$
\mathrm{J}-\mathrm{X}_{21}, \quad \mathrm{~J}-\mathrm{X}_{30}, \quad \mathrm{~J}-\mathrm{X}_{45}, \quad \mathrm{~J}-\mathrm{X}_{60} ;
$$

- sur les valeurs relatives d'autre part (p. IOo);

$$
\frac{J-X_{21}}{J}, \quad \frac{J-X_{30}}{J}, \quad \frac{J-X_{45}}{J}, \quad \frac{J-X_{60}}{J} .
$$

\section{TABLEAU III}

Moyennes et écarts-types des différences

\begin{tabular}{|c|c|c|c|c|c|c|c|c|}
\hline \multirow[b]{2}{*}{ Périodicité du Contrôle } & \multicolumn{2}{|c|}{$2 \mathrm{I}$ jours } & \multicolumn{2}{|c|}{ 3o jours } & \multicolumn{2}{|c|}{45 jours } & \multicolumn{2}{|c|}{60 jours } \\
\hline & $\mathrm{J}-\mathrm{X}_{21}$ & $\frac{\mathrm{J}-\mathrm{X}_{21}}{\mathrm{~J}}$ & $\mathrm{~J}-\mathrm{X}_{30}$ & $\frac{\mathrm{J}-\mathrm{X}_{30}}{\mathrm{~J}}$ & $J-X_{45}$ & $\frac{J-X_{45}}{J} \mid$ & $J-X_{60}$ & $\frac{J-X_{60}}{J}$ \\
\hline $\begin{array}{l}\text { Moyenne algébrique... } \\
\text { Ecart-ty pe (valeurs } \\
\text { algébriques)........ }\end{array}$ & $\begin{array}{r}+13 \\
109\end{array}$ & $\begin{array}{r}+0,4 \% \\
3, \mathbf{\%} \%\end{array}$ & $\begin{array}{rr}- & \mathrm{r} 6 \\
\mathrm{r} 3 \mathrm{r}\end{array}$ & $\begin{array}{r}-0,5 \% \\
3,7 \%\end{array}$ & $\begin{array}{r}50 \\
188\end{array}$ & $\begin{array}{r}+\quad 1,4 \% \\
5,3 \%\end{array}$ & $\begin{array}{r}-\quad 48 \\
205\end{array}$ & $\begin{array}{r}-\mathrm{r}, 4 \% \\
5,8 \%\end{array}$ \\
\hline Moyenne arithmétique.. & 85 & $2,4 \%$ & 94 & $2,6 \%$ & I33 & $\begin{array}{ll}3,5 & 70 \\
3,7 & \%\end{array}$ & I69 & $4,7 \%$ \\
\hline
\end{tabular}
en valeurs absolues $(k g)$ et en valeurs relatives ( $p$. IOo).

Enfin, dans le cas d'un contrôle mensuel uniquement, nous avons vérifié que la précision ainsi calculée était identique à celle qui ressortait de l'application de la méthode dite $\mathrm{D}$. La dispersion des écarts obtenus dans le dernier cas (écart-type des différences algébriques par rapport au contrôle journalier) est même légèrement inférieure (tableau IV) à celle due à l'application de la méthode Fleischmann.

TABLEAU IV

Différences entre les productions réelles et les productions contrôlées

\begin{tabular}{|c|c|c|}
\hline & $\mathrm{J}-\mathrm{D}_{30}(\mathrm{~kg})$ & $\frac{J-D_{30}}{J}(\%)$ \\
\hline 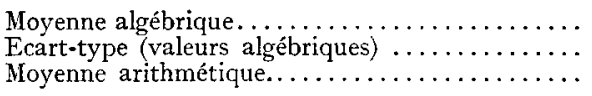 & $\begin{array}{c}+5,6 \\
125 \\
88,6\end{array}$ & $\begin{array}{r}0,43 \\
3,45 \\
2,49\end{array}$ \\
\hline
\end{tabular}
selon la méthode dite $D$.

\section{4,2. - Discussion.}

On peut tirer de ces résultats un certain nombre de conclusions.

4,2.I. - Les méthodes de contrôle laitier basées sur un échantillonnage périodique fournissent un résultat de production ne différant pas statistiquement de la performance réelle; les erreurs moyennes algébriques sont en effet voisines de zéro. 
4,2.2. - L'incertitude statistique sur la production laitière qui peut être définie par l'erreur attachée au seuil de probabilité de 5 p. Ioo et qui est égale au double de l'éart-type varie entre 6,2 p. roo (périodicité de 2 I jours) et Ir,6 p. Ioo (périodicité de 6 semaines). Elle augmente

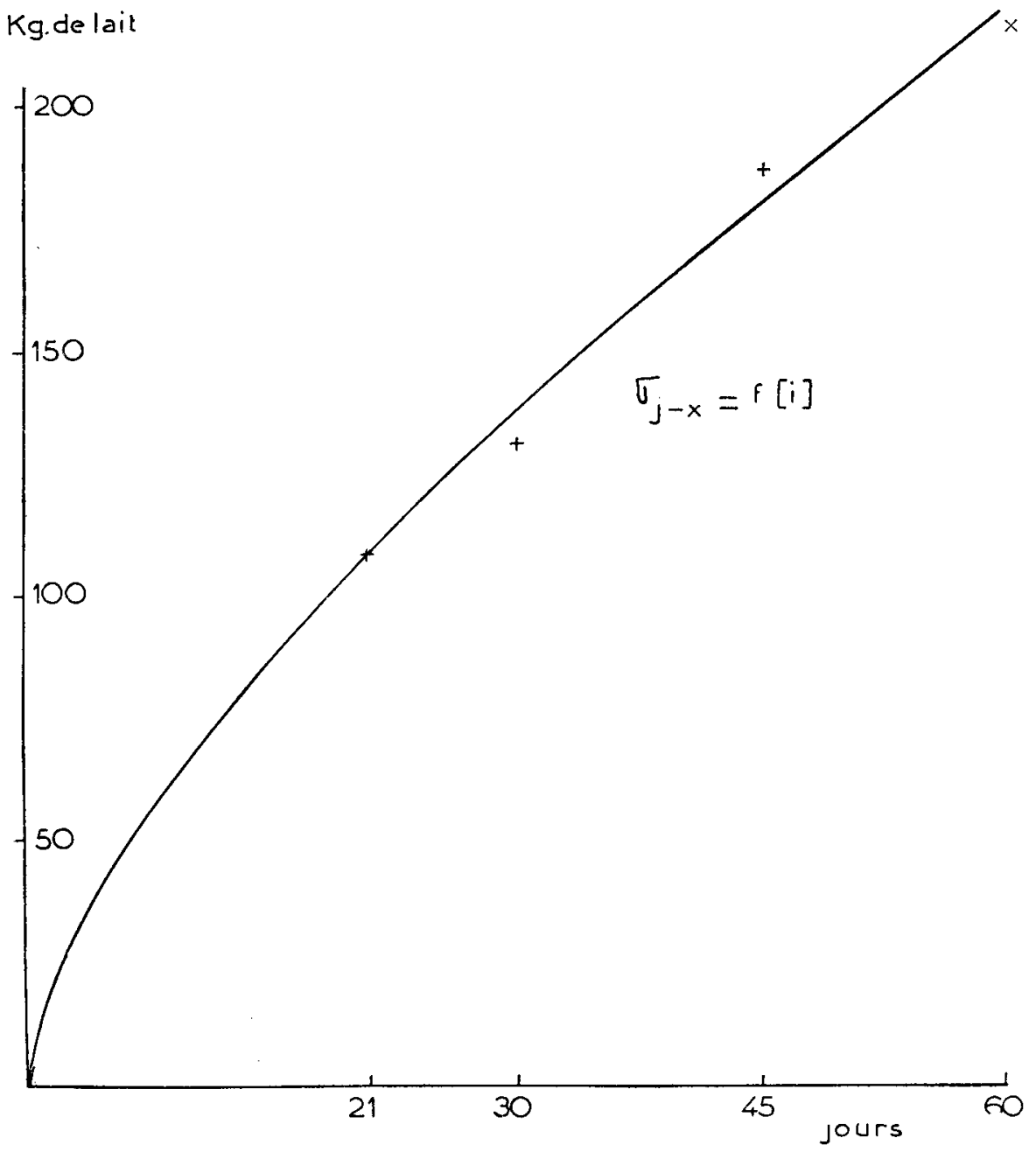

FIG. I. - Écart-type des erreurts en fonction de la périodicité du contrôle.

avec la durée de 1'intervalle moyen entre les contrôles; toutefois, nous ne possédons pas suffisamment de points caractéristiques pour étudier l'expression mathématique de la courbe représentative de cette relation (fig. I). Dick (7) a montré, à ce sujet, que l'écart-type des erreurs était lié à la durée moyenne de l'intervalle de contrôle par une loi linéaire. Dans l'ensemble, les différences arithmétiques que nous obtenons 
sont plus élevées que celles qui sont signalées par d'autres auteurs. Ainsi, MacCARThy et Boyle (27) qui ont étudié la précision d'un contrôle laitier à périodicité variable trouvent les valeurs suivantes pour les écartstypes des différences entre la production réelle et la performance correspondant à un contrôle tous les 30,45 et 60 jours : 3, I p. Ioo dans le cas du contrôle mensuel (3,7 p. Ioo dans notre étude), 4,7 p. Ioo dans le cas d'un contrôle toutes les 6 semaines (5,3 p. Ioo dans notre étude), et 5,4 dans le cas d'un contrôle bimestriel $(5,8 \mathrm{p}$. Ioo dans notre étude). Dick (7), M'Candish et coll. (26), Ashton (2) qui étudient la précision du contrôle mensuel fournissent d'ailleurs des chiffres encore plus faibles : 2,39 p. IOO, 2,00 p. Ioo et I,70 p. Ioo respectivement. Il est vraisemblable de penser que cette discordance entre nos résultats et ceux des chercheurs étrangers est due, pour une part, à des différences dans les conditions d'entretien des vaches laitières. Alors que les données généralement utilisées par les auteurs proviennent de stations de recherches où les animaux sont bien alimentés et dans un bon état sanitaire, le troupeau de La Pierre-qui-vire a dî subir, au cours de la période sur laquelle a porté cette étude, plusieurs disettes (en hiver notamment) ; la présence de mammites chroniques chez la plupart des vaches a dû, par ailleurs, contribuer à accroître la variabilité journalière de la production et peut être considérée comme une cause d'erreur appréciable.

4,2.3. - Connaissant la précision de l'estimée de la performance individuelle, à partir des résultats d'un contrôle laitier périodique, il est facile de déterminer l'erreur que l'on commet en appréciant par la même méthode d'échantillonnage la moyenne d'un groupe de lactations dans le cas du testage par exemple. On sait, en effet, que l'écart-type d'une moyenne de résultats $(\overline{\mathrm{X}})$ se déduit de l'écart-type des données individuelles $(\mathrm{X})$ par la relation :

$$
\sigma_{x}=\frac{\sigma_{x}}{\sqrt{n}}
$$

$n=$ nombre de valeurs de $\mathrm{X}$ utilisées pour calculer $\overline{\mathrm{X}}$.

Nous avons représenté, dans le tableau $V$, les valeurs de l'incertitude statistique attachée au calcul d'une moyenne de performances laitières pour diverses périodicités du contrôle : 2I, 30, 45 et 60 jours. Ce travail a également été réalisé par ERB et coll. (10), qui trouvent des chiffres sensiblement plus faibles que les nôtres.

On remarque à l'examen de ces chiffres qu'une production individuelle est connue par un contrôle mensuel avec la même précision que la moyenne de 2 à 3 performances contrôlées tous les 2 mois. Sur une plus vaste échelle, l'erreur que l'on commet dans l'estimation d'une moyenne de 9 lactations contrôlées tous les mois est identique à celle qui correspond à un groupe de 25 lactations contrôlées tous les 2 mois. Si 
on se réfère donc à la seule précision de l'estimée d'une moyenne de performances, il n'est donc pas rentable de porter de I mois à 2 mois la durée de l'intervalle de contrôles. Cela reviendrait, en matière de testage par exemple, à contrôler moins de filles mais d'une façon plus précise On sait, par contre, que pour diminuer la part due au milieu dans le comparaison de la production de lots de filles, il faut prévoir des lots suffisamment importants de génisses. Aucune étude systématique envisageant la périodicité optimum de contrôle à prévoir dans la conduite d'une opération de testage n'existe à notre connaissance. Ashron (2), ERB et coll. (IO) pensent, comme nous, qu'un contrôle à périodicité plus large que le contrôle mensuel suffirait à exprimer la production des filles d'un lot de testage. Une étude sur ce point sera entreprise ultérieurement.

TABLEAU V

Incertitude statistique sur l'estimée d'une moyenne de performances (en $\mathrm{kg}$ de lait).

\begin{tabular}{|c|c|c|c|c|c|c|c|c|c|}
\hline \multirow{2}{*}{$\begin{array}{c}\text { Périodicité } \\
\text { des contrôles (en jours) }\end{array}$} & \multicolumn{9}{|c|}{ Nombre de filles } \\
\hline & I & 2 & 3 & 4 & 9 & 16 & 25 & $3^{6}$ & 49 \\
\hline $2 I$ & 218 & 156 & I 28 & 109 & 93 & 54 & 43 & $3^{6}$ & $3^{I}$ \\
\hline 30 & 262 & 187 & I 54 & ${ }^{3} 3 \mathrm{I}$ & 87 & 65 & 54 & 44 & 37 \\
\hline 45 & 376 & 268 & 201 & 183 & 125 & 94 & 75 & 63 & 53 \\
\hline 60 & 410 & 292 & $24 \mathrm{I}$ & 205 & 137 & 102 & 82 & 68 & 58 \\
\hline
\end{tabular}

4,2.4. - On a pu enfin calculer la précision d'un contrôle laitier bimestriel par rapport au contrôle mensuel dont l'étude va justement faire l'objet de la $2^{\mathrm{e}}$ partie de notre travail.

On a, en effet:

$$
\begin{aligned}
\frac{X_{60}-X_{30}}{J} & =\frac{J-X_{30}}{J}-\frac{J-X_{60}}{J} \\
\sigma^{2}\left(\frac{X_{60}-x_{30}}{J}\right) & =\sigma^{2}\left(\frac{J-X_{30}}{J}\right)+2^{2}\left(\frac{J-X_{60}}{J}\right)-2 \operatorname{cov} \cdot\left(\frac{J-X_{30}}{J}, \frac{J-X_{60}}{J}\right) \\
& =(3,7)^{2}+(5,8)^{2}-2(0,03)(3,7)(5,8) \\
\sigma\left(\frac{x_{60}-X_{30}}{J}\right) & =6,78 \% .
\end{aligned}
$$

\section{V. - DEUXIÈME ÉTUDE. DONNÉES DU SYNDICAT DE LA MANCHE}

5,1. - A partir de l'échantillon de données du Syndicat de la Manche, nous avons calculé, pour chaque lactation, les productions de lait, de matière grasse et le taux butyreux, d'après le principe de la méthode dite $\mathrm{D}$, en utilisant : 
Io Les résultats du contrôle mensuel (D).

$2^{\circ}$ Les résultats d'un contrôle bimestriel fictif obtenus en retenant:

- soit les contrôles pairs $\left(\mathrm{D}_{2 p}\right)$,

- soit les contrôles impairs $\left(\mathrm{D}_{2 i_{i}}\right)$.

La méthode $\mathrm{D}$ que nous avons définie par ailleurs pour le contrôle mensuel prend, dans le cas d'un échantillonnage bimestriel, la forme particulière stivante :

$$
\mathrm{D}_{2}=\frac{\Sigma c}{n}(\mathrm{~F}+30-\mathrm{N})+\mathrm{c}_{1}(\mathrm{~N}-30) .
$$

$\Sigma c=$ somme des productions de lait ou de matière grasse enregistrées au cours des contrôles.

$\mathrm{N}=$ intervalle vêlage-premier contrôle.

$\mathrm{F}=$ durée de lactation d'après la méthode Fleischmann.

$n=$ nombre de contrôles.

Les lactations présentant un écart entre deux contróles successifs supérieur à 50 jours (contrôle manquant) ont été éliminées de cette étude ; cela ne saurait diminuer en rien la validité des résultats obtenus puisque, la méthode dite $\mathrm{D}$, comme la méthode Fleischmann d'ailleurs, suppose que les contrôles sautés sont remplacés par des valeurs fictives obtenues en faisant la moyenne arithmétique des résultats du contrôle précédent et du contrôle suivant. Les données brutes obtenues par voie mécanographique ont dû être vérifiées dans le but d'éliminer les erreurs inévitables tant dans la perforation des données que dans 1'exploitation des cartes perforées.

Notre étude a porté principalement sur la précision du contrôle bimestriel dans l'estimation de la production laitière totale; les recherches correspondantes sur le taux butyreux et la production de matières grasses ont été limitées à un petit échantillon de données. Nous avons envisagé successivement :

- l'étude statistique générale des populations de différences entre la production obtenue par un contrôle tous les deux mois (contrôles pairs et contrôles impairs séparément) et la performance calculée à partir d'un contrôle mensuel.

- les causes de variations systématiques des différences (en p. IOo), leur élimination physique ou statistique et l'intérêt que présente cette élimination pour améliorer la précision du contrôle bimestriel.

\section{5,2. - Étude statistique générale des populations de différences.}

Les paramètres statistiques des populations de différences (en p. Ioo) entre ces 2 méthodes d'échantillonnage périodique ont été calculés: 
a) Pour la production laitière totale de la lactation sur l'ensemble des données (tableau VI).

\section{Tableau VI}

Paramètres statistiques des populations de différences $\frac{\mathrm{D}_{1}-\mathrm{D}_{2}}{\mathrm{D}_{1}}$ (production laitière).

\begin{tabular}{|c|c|c|c|}
\hline & $\frac{\mathrm{D}_{1}-\mathrm{D}_{2 p}}{\mathrm{D}_{1}}$ & $\frac{D_{1}-D_{2 i p}}{D_{1}}$ & Totalité des différences \\
\hline $\begin{array}{c}\text { Nombre de données...... } \\
\text { Différence moyenne (valeur } \\
\text { arithmétique)......... } \\
\text { Différence moyenne (valeur } \\
\text { algébrique) } \ldots \ldots \ldots \ldots \ldots \\
\text { Ecart-type } \ldots \ldots \ldots \ldots \ldots \ldots \\
\text { Skewness } \ldots \ldots \ldots \ldots \ldots \ldots \ldots \\
\text { Kurtosis } \ldots \ldots \ldots \ldots \ldots \ldots\end{array}$ & $\begin{array}{l}1990 \\
3,93 \% \\
+0,61 \% \\
4,77 \% \\
+0,234 \\
1,04\end{array}$ & $\begin{array}{l}1990 \\
3,28 \% \\
-0,31 \% \\
-3,96 \% \\
-0,022 \\
\mathbf{1 , 7 0}\end{array}$ & $\begin{array}{r}+0,19 \% \\
4,40 \%\end{array}$ \\
\hline
\end{tabular}

b) Pour les productions de lait, de matière grasse et le taux butyreux sur un échantillon réduit de valeurs (tableau VII).

\section{TABLEAU VII}

Paramètres statistiques des populations de différences sur petit échantillon (production laitière, production de matière grasse, taux butyreux).

\begin{tabular}{|c|c|c|c|c|c|c|c|c|c|}
\hline \multirow{3}{*}{$\frac{\text { Nombre de données...... }}{\text { Performance considérée.. }}$} & \multicolumn{3}{|c|}{$\frac{\mathrm{D}_{1}-\mathrm{D}_{2 p}}{\mathrm{D}_{1}}$} & \multicolumn{3}{|c|}{$\frac{\mathrm{D}_{1}-\mathrm{D}_{\mathbf{2} i p}}{\mathrm{D}_{1}}$} & \multicolumn{3}{|c|}{ Totalité des différences } \\
\hline & \multicolumn{3}{|c|}{235} & \multicolumn{3}{|c|}{235} & \multicolumn{3}{|c|}{470} \\
\hline & P. L. & M. G. & T. B. & P. L. & M. G. & T. B. & P. L. & M. G. & T. B. \\
\hline $\begin{array}{l}\text { Moy. algébrique }(\%) . . . \\
\text { Moy. arithmétique }(\%) . . \\
\text { Ecart-type }(\%) \ldots \ldots \ldots \\
\% \text { d'erreurs sup. à } 5 \% . \\
\% \text { d'erreurs sup. à } 10 \% .\end{array}$ & $\mid \begin{array}{r}0,05 \\
3,01 \\
4,06 \\
17,4 \\
3,0\end{array}$ & $\begin{array}{r}1,83 \\
4,69 \\
5,87 \\
3^{8,6} \\
14,5\end{array}$ & $\begin{array}{c}1,92 \\
3,36 \\
4, \mathrm{II} \\
26,5 \\
4,3\end{array}$ & $\begin{array}{c}+0,12 \\
3,76 \\
4,9 \mathrm{r} \\
26,5 \\
6,4\end{array}$ & $\begin{array}{r}\mathrm{I}, \mathrm{I} 5 \\
5,22 \\
6,75 \\
40,6 \\
\mathrm{I} 4, \mathrm{I}\end{array}$ & $\begin{array}{c}\mathrm{I}, 60 \\
3,84 \\
4,83 \\
30,4 \\
7,8\end{array}$ & $\begin{array}{r}+0,09 \\
3,38 \\
4,50 \\
22,0 \\
4,7\end{array}$ & $\mid \begin{array}{r}1,49 \\
4,96 \\
6,28 \\
39,6 \\
14,3\end{array}$ & $\begin{array}{r}\mathrm{I}, 76 \\
3,60 \\
4,48 \\
28,5 \\
6, \mathrm{I}\end{array}$ \\
\hline
\end{tabular}

Nous avons considéré séparément, dans chaque cas, les différences associées aux contrôles pairs, aux contrôles impairs et à la totalité des données ; cette dernière répartition correspond en fait aux résultats que l'on obtient dans les conditions pratiques d'application du contrôle bimestriel; le premier contrôle après la mise-bas peut, en effet, tomber aussi bien dans le premier (cas des contrôles impairs) que dans le second (cas des contrôles pairs) mois de lactation.

Le fait que la moyenne algébrique des différences dans le cas de la production laitière (échantillon global) est positive quand on considère les contrôles pairs $(+0,6 \mathrm{r}$ p. roo) et négative lorsqu'on se sert des contrôles impairs (- o, $3^{I}$ p. IOO) conduit à penser qu'il existe une différence 
systématique entre les résultats fournis par ces 2 méthodes de détermination de la production. Il est en effet logique de supposer que la position du premier contrôle par rapport au maximum de la courbe de lactation fixe en partie le sens et l'importance de l'erreur commise sur l'estimation de la production laitière. Lorsque ce contrôle se situe, par exemple, dans le deuxième mois de lactation, on risque, dans la plupart des cas, de ne pas rendre compte de la "pointe de lait " après vêlage ; cela explique, selon nous, l'erreur moyenne par défaut que l'on obtient alors ( $-0,3$ I p. IOO). Cette question fera d'ailleurs l'objet d'une étude plus détaillée.

I,es écarts-types des différences calculées pour le taux butyreux sont voisins de ceux qui apparaissent dans le cas de la production laitière. En ce qui concerne la matière grasse, les erreurs semblent un peu plus élevées (Io) ; la précision de l'estimée de ce critère dépend, en effet, de celle de la production laitière et de celle du taux butyreux. Une étude systématique des variations de jour à jour de la quantité de lait produite pourra nous éclairer sur ce point.

On remarquera, par ailleurs, que les écarts-types des différences obtenus pour la production laitière par lactation (respectivement de $4,4 \%$ et $4,48 \%$ pour les contrôles d'ordre pair et impair.) sont sensiblement plus faibles que celui auquel le calcul a abouti sur les données de l'élevage de la Pierre-qui-Vire $(6,78 \%)$. Cette différence peut s'expliquer par le fait que des erreurs absolues de même grandeur dans les 2 populations ont été rapportées dans le $2^{\text {eme }}$ cas à des productions laitières en moyenne plus faibles que celles observées dans le département de la Manche, ce qui a évidemment pour conséquence logique d'entraîner un accroissement sensible des erreurs relatives dans l'estimation des perfomances à partir d'un contrôle de périodicité mensuelle par rapport à celle que l'on obtient à partir d'un contrôle de périodicité bimestrielle.

\section{5,3. - Causes de variation des dijiéérences. Production laitière.}

Après avoir étudié la population générale de différences entre la production laitière d'après un contrôle bimestriel et la même performance à partir d'un contrôle mensuel, il importe de déterminer les causes de variation de ces différences et d'essayer de les réduire dans la pratique d'un contrôle bimestriel. Nous avons envisagé 3 sources d'erreur principales :

(I) la durée de l'intervalle entre le vêlage et le premier contrôle ; nous avons vu que la position de ce dernier, par rapport au maximum de production peut introduire une erreur systématique,

(2) le niveau de production laitière,

(3) la durée de la lactation dont l'influence sur la précision du con- 
trôle bimestriel par rapport au contrôle mensuel revêt un double aspect :

(a) la durée du cycle de production fixe le nombre de contrôles utilisables pour le calcul de la production totale donc l'importance de l'erreur statistique attachée à la connaissance de la performance de production,

(b) la durée réelle de lactation est moins bien connue avec les contrôles bimestriels car on utilise une durée de tarissement systématique de 30 jours.

\section{5,3.1. - Durée de l'intervalle vêlage-premier contrôle.}

Pour 1'étude de ce facteur, les différences algébriques en valeur relative $\frac{\mathrm{D}_{1}-\mathrm{D}_{2}}{\mathrm{D}_{1}}$ ont été groupées par classes de 5 jours d'intervalle vêlage-premier contrôle. La variation des différences moyennes par classe est représentée par un graphique (fig. 2) ; elle s'établit suivant une courbe

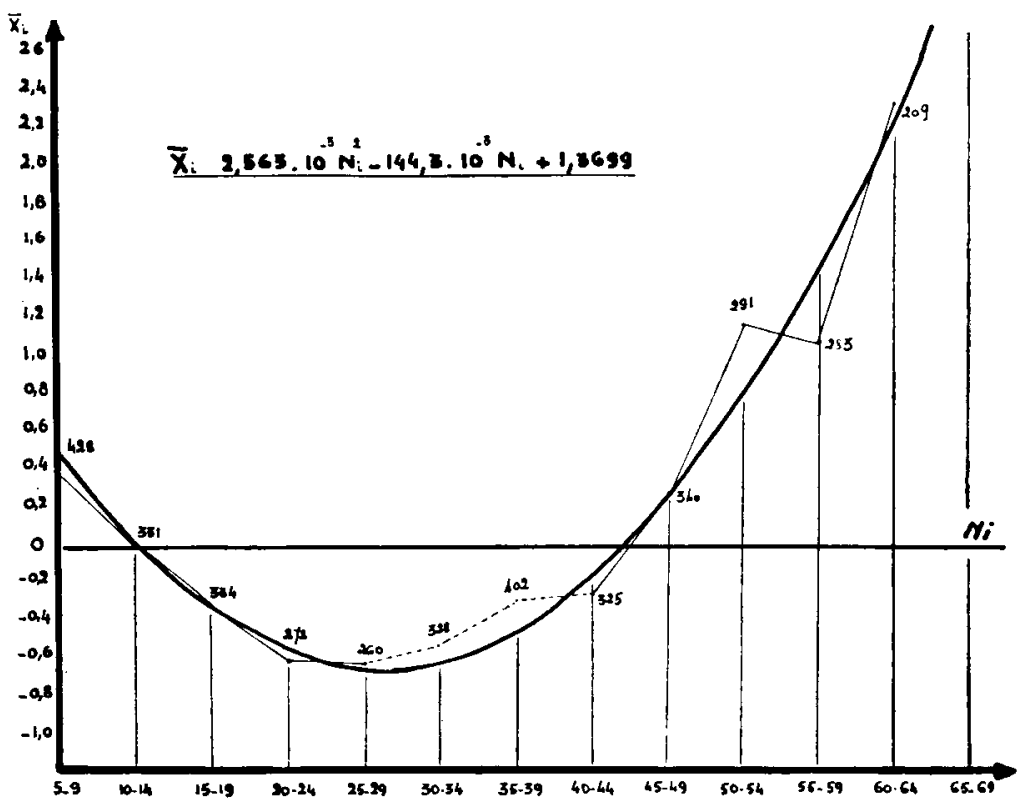

IIG. 2. - Variation de la moyenne algébrique des différences entre des lactations calculées selon des contrôles mensuels et bimestriels, groupées par classes de dix jours d'intervalle vêlage-premier contrôle.

d'allure parabolique qui s'apparente logiquement à la courbe moyenne de lactation des vaches laitières dans le département de la Manche. L orsque le premier contrôle se situe vers $27-28$ jours après le vêlage, on détermine en général une valeur maximum de la production ; cette position du premier contrôle correspond donc au sommet de la courbe de 
lactation. Par contre, comme nous l'avons déjà signalé, quand ce premier contrôle se situe au-delà de $1 \mathrm{a} 6^{\mathrm{e}}$ semaine après la mise-bas, on ne rend pas assez compte de la " pointe de lait » et on obtient une valeur par défaut qui est d'autant plus erronée que l'intervalle vêlage-premier contrôle est long. Il convient de préciser que cette interprétation n'est valable qu'à l'échelon d'une population comme celle que nous étudions, car il existe évidemment des différences importantes dans la forme de la courbe de lactation de chaque vache et en particulier dans la position du maximum de cette courbe par rapport au vêlage.

L'équation de régression parabolique des différences $\frac{D_{1}-D_{2}}{D_{2}}$ sur les valeurs de l'intervalle mise-bas-premier contrôle a été calculée dans le but de déterminer des facteurs de correction de la production laitière pour chaque classe et de réduire statistiquement l'importance de cette cause d'erreur.

Si nous désignons par Nil'intervalle moyen correspondant à la classe $i$ et par $\overline{\mathrm{X}}_{i}$ la différence moyenne par classe, qui représente le facteur de correction en $\mathrm{p}$. Ioo de la production laitière, l'équation de régression s'écrit :

$$
\overline{\mathrm{X}}_{i}=2,563 . \quad \mathrm{I0}^{-3} \mathrm{~N}_{i}^{2}-\mathrm{I} 44,3 . \quad \mathrm{I0}^{-3} \mathrm{~N} i+\mathrm{I}, 3699 .
$$

$\mathrm{L}$ a valeur minimum de $\overline{\mathrm{X}_{i}}$ est obtenue pour $\mathrm{N}_{i}=\frac{\mathrm{I} 44,3}{2 \times 2,563}=28$, I jours

Cette correction diminue de 18 à 20 p. Ioo 1'erreur commise quand on utilise le contrôle bimestriel à la place du contrôle mensuel. Elle s'impose donc dans ce cas ; nous pensons également qu'elle pourrait s'appliquer au calcul mécanographique des lactations contrôlées tous les mois, tout au moins lorsque le premier contrôle est situé trop près (moins de ro jours) ou trop loin (plus de 40 jours) du vêlage.

\section{5,3.2. - Niveau de production laitière.}

La dispersion des différences entre la production laitière d'après un contrôle bimestriel et cette même performance obtenue à partir d'un contrôle mensuel a été étudiée en fonction du niveau de production laitière de la femelle exprimée par la production totale de la lactation. Nous avons caractérisé cette dispersion par:

- la différence moyenne arithmétique, $\left|\overline{\mathrm{X}_{p}}\right|$ ou $\left|\overline{\mathrm{X}_{i p}}\right|$ (fig. 3),

- l'écart-type des différences ${ }^{5} \mathrm{x}_{p}$ et ${ }^{\sigma} \mathrm{X}_{i p}$ (fig. 4).

Ces 2 paramètres ont été calculés par classes de $500 \mathrm{~kg}$ de production laitière $\left(D_{1}\right)$; leurs variations sont représentées graphiquement (fig. 3 et 4); nous avons déterminé par ailleurs les coefficients? de corrélation 


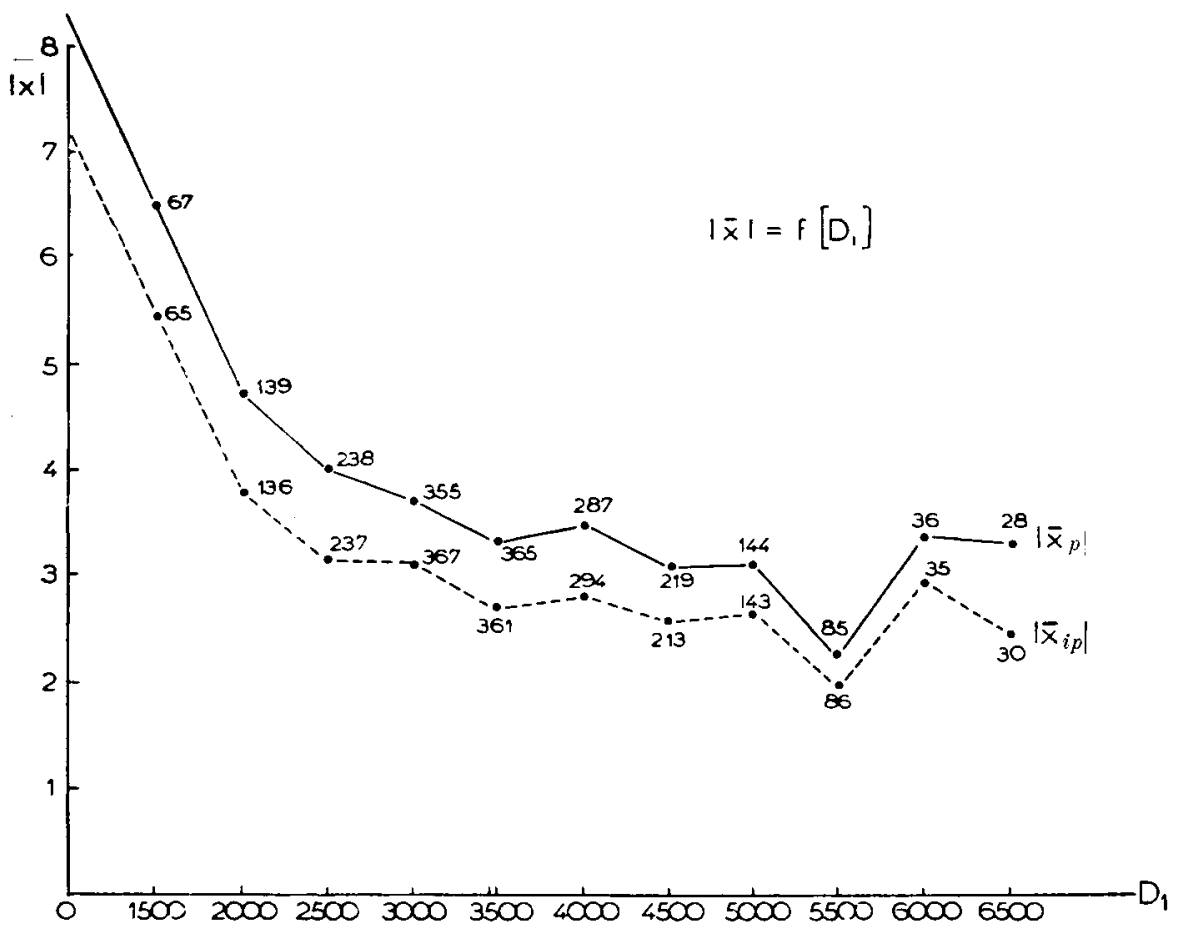

FIG. 3. - Variation de la moyenne arithmétique des différences entre des lactations calculées selon des contrôles mensuels et bimestriels, groupées par classes de $500 \mathrm{~kg}$ de niveau de production laitière.

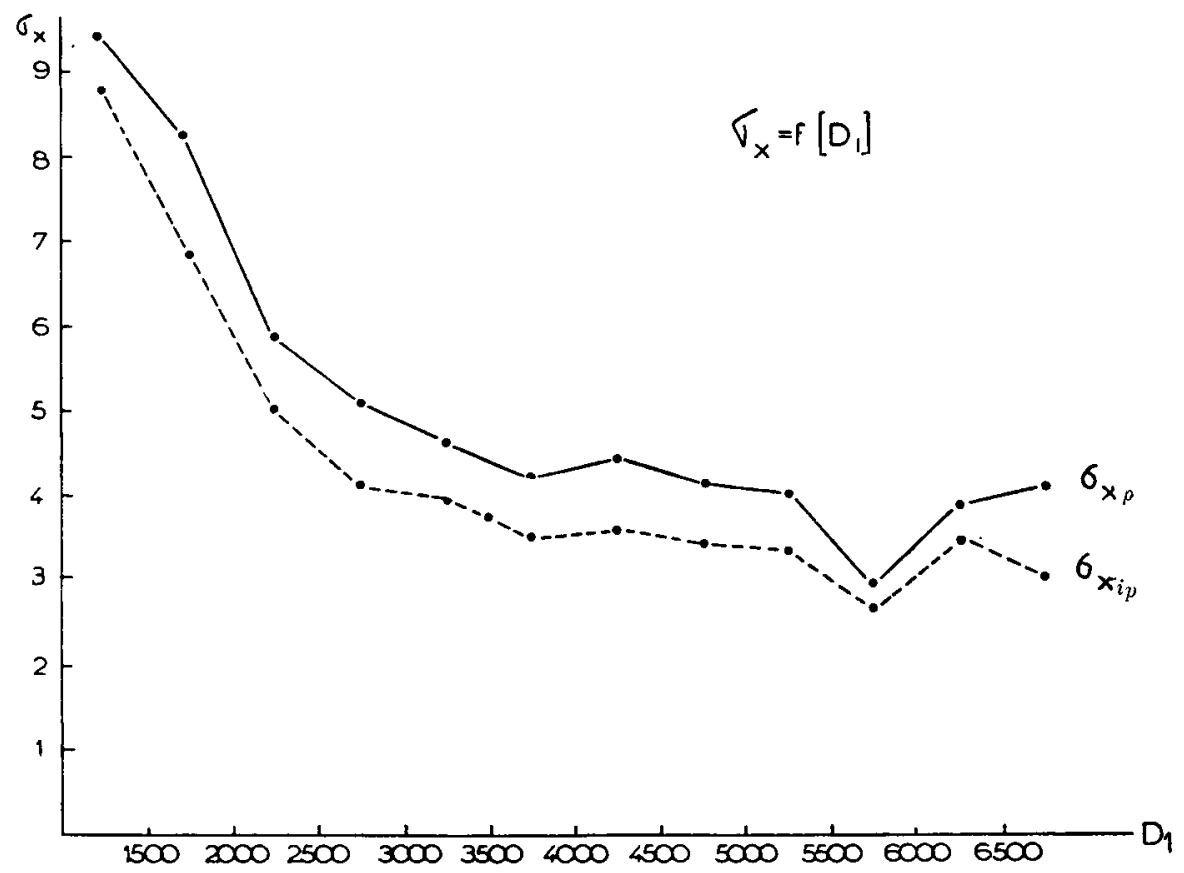

FIG. 4. - Variation de l'écart-type des différences algébriques entre des lactations calculées selon des contrôles mensuels $e t$ bimestriels, sroupées par classes de $500 \mathrm{~kg}$ de niveau de production laitière. 
linéaire entre le niveau de production moyen de chaque classe et des valeurs correspondantes de chacun des 2 critères de dispersion.

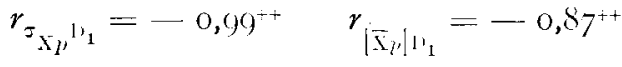

$$
\begin{aligned}
& r_{\sigma_{\mathrm{X} i}, \mathrm{D}_{1}}=-0,95^{++} \quad r_{|\overline{\mid \bar{i} i}|_{\mathrm{I}_{1}}}=-0,84^{++} \text {. }
\end{aligned}
$$

On remarque, à l'examen de ces graphiques, que les moyennes et écarts-types sont sensiblement constants au-delà de $3000 \mathrm{~kg}$ de lait. Les productions inférieures à ce seuil donnent lieu, par contre, à des différences souvent plus importantes; elles correspondent, pour la plupart, à des performances réalisées au cours de lactations incomplètes; c'est donc, en fait, à une influence propre de la durée de lactation qu'on a affaire dans ce cas. Il ne semble pas, en définitive, que le niveau de production laitière des femelles intervienne dans la répartition statistique des différences entre la production d'après un contrôle bimestriel et la production à partir d'un contrôle mensuel.

\section{5,3.3. - Durée de la lactation.}

La précision statistique de détermination de la production laitière totale à partir d'un contrôle périodique dépend en partie du nombre de résultats de contrôle utilisables. Flle est donc directement fonction de la durée de lactation.

$I^{0}$ Nous avons étudié cette question dans le cadre de la comparaison des contrôles mensuels et binestriels en calculant, par classes de 30 jours de durée de lactation, la différence moyenne arithmétique et l'écart-type des différences. Les courbes de variation de ces valeurs ont été tracées (fig. 5 et 6); elles présentent une allure identique à celle que nous avons établie dans l'étude du niveau de production laitière. Cette remarque confirmerait l'idée que nous avons émise selon laquelle l'action de ce facteur se manifesterait surtout par une variation de la durée de lactation avec la production laitière.

On remarque, à l'examen des courbes que la durée du cycle de production n'agit que faiblement sur la précision du contrôle bimestriel au-delà de 240 jours. I,es coefficients de régression obtenus au-delà de cette limite sont négatifs et très faibles. Par contre, le contrôle bimestriel peut s'accompagner d'erreurs importantes lorsque les lactations ont une durée inférieure à 240 jours; nous pensons que, dans la pratique du testage basé sur un contrôle bimestriel, de telles lactations devraient être éliminées.

Dans cet esprit, nous avons calculé l'incidence de cette suppression des lactations courtes dans notre échantillon de travail sur la précision du contrôle bimestriel par rapport au contrôle mensuel. Pour 3890 lactations, l'élimination a porté sur 336 données, soit $8,5 \mathrm{p}$. Ioo du total. 


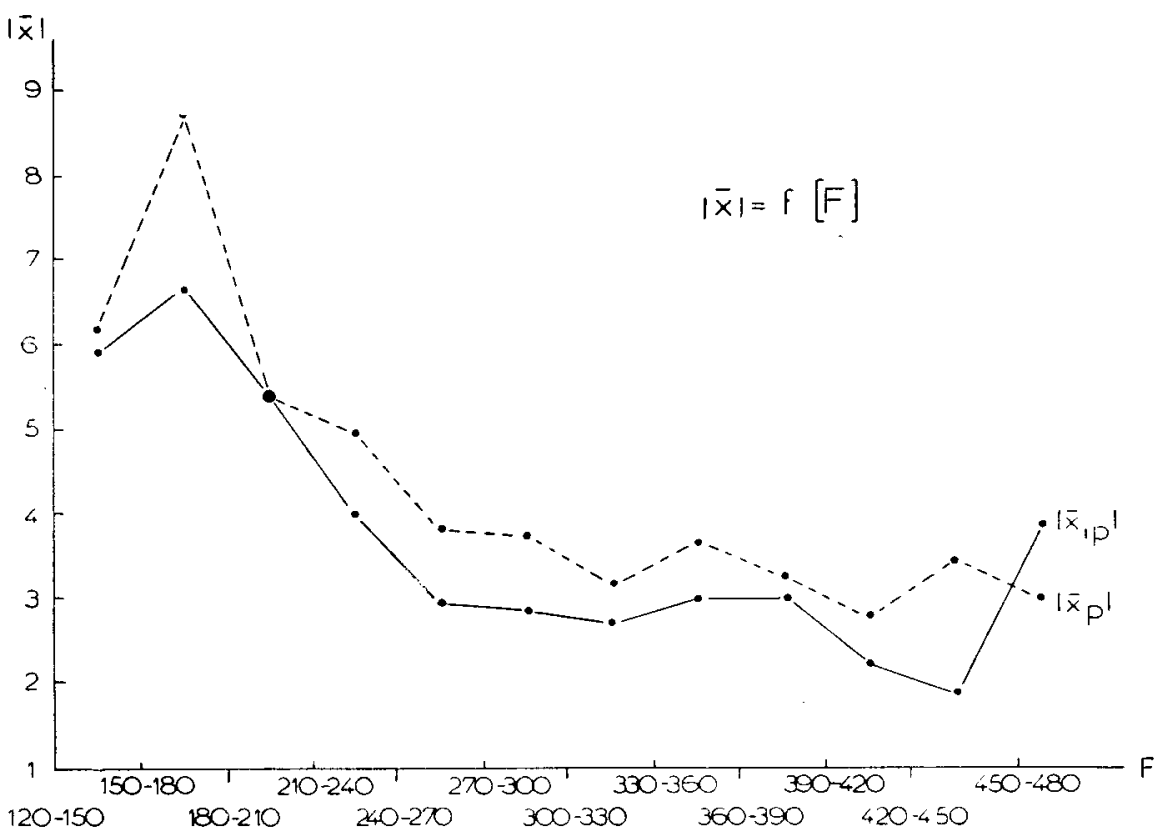

FIG. 5. - Variation de la moyenne arithmétique des différences entre des lactations calculées selon des contrôles mensuels et bimestriels, groupées par classes de 30 jours de durée de lactation.

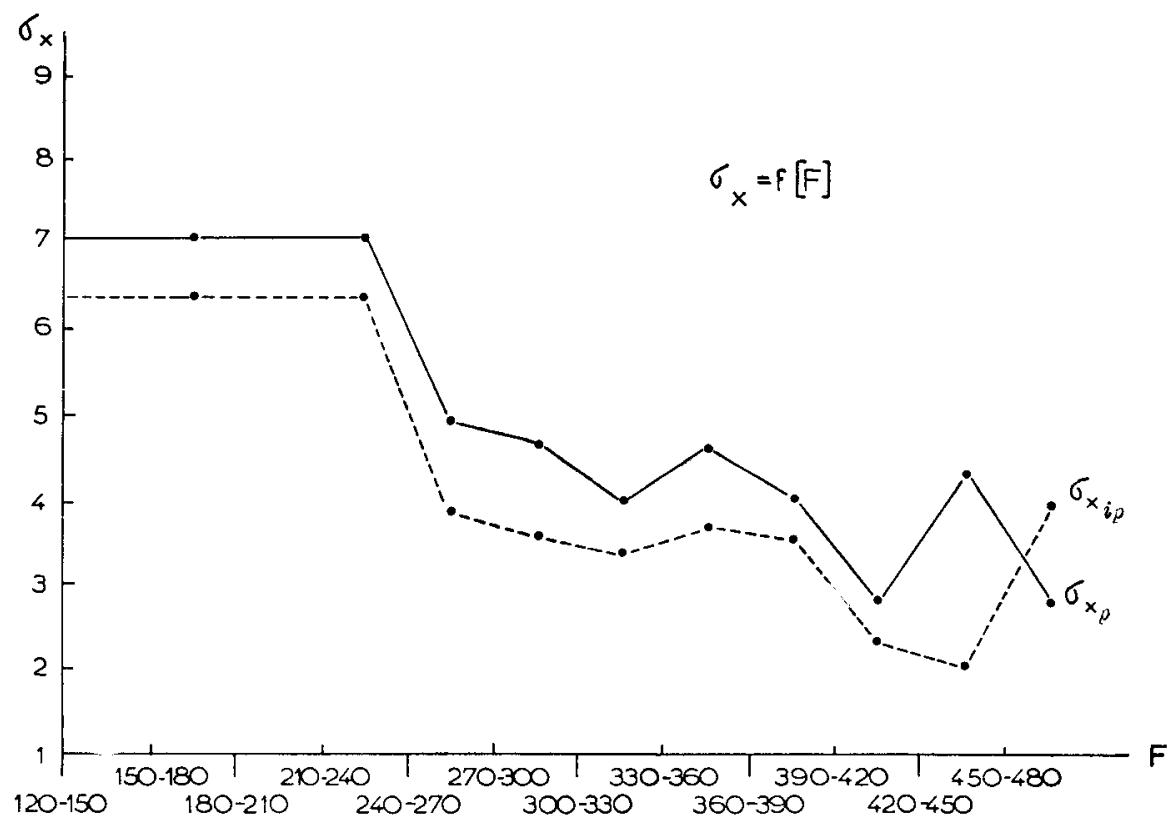

FIG. 6. - Variation de l'écart-type des différences algèbriques entre des lactations calculées selon des contrôles mensuels et bimestriels, groupées par classes de $3^{\circ}$ jours de durée de lactation. 
La moyenne de la nouvelle population de différences arithmétiques est de 3,23 p. Ioo au lieu de 3,47 p. Ioo pour l'ensemble (réduction de $7 \mathrm{p}$. Ioo). L'écart-type de la population de valeurs algébriques devient 4,I7 p. I0O au lieu de 4,50 p. Ioo (diminution de 7 p. Ioo également).

$2^{\circ}$ Nous n'avons pas étudié de façon rigoureuse l'erreur que l'on commet par un contrôle bimestriel, par rapport au contrôle mensuel, sur l'appréciation de la date du tarissement. Cette recherche statistique est compliquée du fait que les populations de différences que l'on envisage dans ce cas ne se répartissent pas normalement; on peut néanmoins avoir une idée de 1'ordre de grandeur de cette imprécision. La durée de lactation est connue, soit par excès, soit par défaut, avec une erreur de I5 jours $\pm \mathrm{e}$, qui est évidemment d'autant plus importante, en valeur relative, que 1'on a affaire à des lactations courtes; (c'est l'écart entre la durée réelle de lactation, et la durée estimée à partir d'un contrôle mensue1). Cette remarque explique 1'allure hyperbolique des courbes correspondant aux graphiques 5 et 6 . L'erreur sur la durée de lactation qui se répercute sur la production laitière serait ainsi, pour le contrôle bimestriel, supérieure d'au maximum 5 p. Ioo $\left(\frac{\mathrm{I}}{300}\right)$, à celle qui correspond à un contrôle mensuel, car la production journalière de la femelle avant son tarissement est en effet inférieure à sa production journalière moyenne. En réalité cette erreur, sans être négligeałle, doit être notoirement plus faible.

Nous pensons qu'il doit être possible de supprimer cette source d'erreurs par une connaissance plus exacte de la date du tarissement :

- d'après les renseignements fournis par l'éleveur, renseignements qui seront d'autant plus justes que ce type de contrôle sera seulement destiné au testage et ne donnera pas lieu à l'élaboration d'un certificat de production ayant une valeur commerciale;

- par, éventuellement, une visite de vérification entre deux contrôles bimestriels successifs.

On pourrait enfin réaliser un contrôle mensuel qui ne porterait que sur une seule traite, alternativement celle $d u$ matin et celle $d u$ soir et qui (3I) équivaudrait en fait à un contrôle bimestriel dans lequel on éviterait l'imprécision supplémentaire attachée à la détermination de la date de tarissement. D'ailleurs, une étude sommaire de ce type de contrôle nous a justement permis de montrer que sa précision par rapport au contrôle mensuel était supérieure à celle du bimestriel ordinaire.

\section{5,4. - Conclusion.}

L'écart-type des différences entre la production laitière calculée à partir d'un contrôle bimestriel et celle qui est obtenue par un échantil- 
lonnage mensuel est de $4,5^{\circ} \mathrm{p}$. Ioo ; c'est un chiffre sensiblement plus faible que celui que nous avions obtenu sur les données de la Pierre-qui-Vire.

On peut améliorer cette précision :

- en appliquant un facteur de correction déterminé statistiquement en fonction de l'intervalle vêlage-premier contrôle;

- en supprimant les lactations trop courtes dans l'exploitation des résultats; femelle.

- en essayant de mieux connaître la date du tarissement fde la

L'utilisation séparée de chacun de ces procédés devrait conduire à une diminution sensible de l'écart-type des différences entre les 2 types de contrôle.

\section{CONCLUSIONS}

Nous pensons pouvoir dégager de notre étude les conclusions suivantes :

$I^{\circ}$ L'erreur statistique attachée à l'échantillonnage périodique de la production laitière, semble supérieure dans les conditions d'exploitation normales, à l'erreur statistique trouvée dans les troupeaux expérimentaux des stations de recherches zootechniques.

$2^{\circ}$ On peut améliorer la précision d'un contrôle périodique par une meilleure connaissance de la courbe de lactation des animaux.

$3^{\circ}$ La cause majeure de l'incertitude attachée à la connaissance de la performance contrôlée par des épreuves périodiques provient de la variabilité journalière de la production de lait et matière grasse que nous nous proposons d'étudier.

$4^{\circ}$ La précision du contrôle mensuel est à peine suffisante pour estimer une performance individuelle, qui n'est alors connue avec 99 p. Ioo de chance, qu'à $\pm 7 \%$.

$5^{\circ}$ L'utilisation des résultats de contrôle laitier pour estimer la valeur d'un groupe de performances permet d'envisager un contrôle à périodicité supérieure au contrôle mensuel ; le contrôle bimestriel semblerait en particulier d'une précision satisfaisante pour calculer les index de sélection sur la descendance des taurillons d'insémination.

Reçu pour publication le 8 août 1957.

\section{RÉFÉRENCES BIBLIOGRAPHIQUES}

(I) Alexander (M. H.) et YapP (W. W.). - Comparison of methods of estimating milk and fat production in dairy cows. J. Dairy Sci.,I949, vol. $32, n^{0} 7$, p. $62 \mathrm{I}-629$.

(2) Ashton (E. D.). - Milk and butterfat recording. Commonwealth Bureau of Dairy Science, I956, Technical Communication, $n^{\circ} 3$. 
$(3)^{*}$ Backhacs. - Ueber die Ermittlung der Leistungsfähigkeit der Milchkühe (Probemelken und Fettbestimmung). Dtsch. L ${ }^{\prime}$ '. Presse, I892, vol. I9, p. 5 I 9.

(4) CAMPBEIL (I. L.). - The accuracy of various methods of estimating a dairy cow's production of milk and butterfat. Proc. Ann. Conf. N. $Z$. Soc. Anim. Prod., r946, p. 99-104.

(5)* Copeiand (L.). - Monthly and bi-monthly tests. Jersey Bull., I928, 47, p. 73I-732.

(6) Davey, Gillian (P.), Alexander (G. I.). - The accuracy of estimation of a dairy cow's production of milk and butterfat. Qd. J. Agric. Sci., I954, vol. II, no 2 , p. 75-77.

(7) Drck (I. D.). - Some results on the accuracy and the daily variability in milk yields. N.Z.J. Sci. Tech., I950, vol. 32 , sect. A, no I, p. 25-29.

(8) DOEHNer (H.). - Handbuch der Schafzüchtung und Schafhaltung. I954, p. 538 .

(9) Döring (H.). - Ueber die Fehler, die bei Milchleistungsprüfungen auftreten. Ztschr. Tierz. und Züchtungsbiol. I950, Bd. 58, p. 389-409.

(Io) ERb (R. E.), Goodwix (M. M.), McCAW (W. N.), Morrissox (R. A.), SHAW (A. O.). - Lactation studies. VI. Improving the accuracy of longer testing intervals and the accuracy of current methods. Washington Agr. Exp. Sta. Circ. 1953, no 230.

(II)* Farrington (E. H.). - I899. Bull. Cornell A gric. Exp. Sta., I69.

(12) F. E. Z. - Principes élémentaires de la production de l'espèce bovine, 1955. Annexe I. - Accord Européen pour 1'unification des méthodes de contrôle laitier-beurrier, Rome, 5-9 mars I95I.

$(\mathrm{r} 3)^{*}$ Fileischmann (W.). - Untersuchung der Milch von I6 Kühen des in Ostpreussen reingezüchteten holländischen Schlages während der Dauer einer Laktation. $L d w^{\prime} . J b ., \mathbf{I} 89 \mathrm{I}$, vol. 20, Eirg-Bd 2, Berlin.

(I4)* GaERTNER. - "How often should one carry out milk recording? "Dtsch. Landw. Tierz., I92 I, vol. 25, p. 85-87.

(I5) Gifford (W.). - The reliability of bi-monthly tests. J. Dairy Sci., I930, vol. I3, no 2 , p. $8 \mathrm{I}-90$.

(I6) Houston (J.), Hale (R. W.). - 'The errors involved in certain methods of estimating the lactation of milk and butterfat. J. Dairy Res., I932, vol. $4, n^{\circ} \mathrm{I}, \mathrm{p} .37-47$.

(I7) Institut International d'Agriculture. - Le contrôle des vaches laitières. dans le monde. Rome 1935 .

(I8) Johansson (I.). - Untersuchungen über die Methodik der Milchleistungskontrolle. Ztschr. f. Tierz und Züchtungsbiol, I942, vol. 5I, p. 229.

(I9) Jordao (L. P.), Patla Assis (F. de), Medixa (P.), Guaragra (R. N.). Estudo sôbre a periodicidade do contrôle quantitativo da produçao leiteira. Bol. Indùstr. anim., I947, no 9, p. 62-7I.

$(20)^{*}$ KaEPPei (J.). - Einiges über das Probemelken der Kühe. Milchw' Zb. I9I6, 45, p. I6.

(2I)* Kirchner. - Wie oft soll das Probemelken vorgenommen werden? Molk. Ztg., I899, 9, p. 2I3.

(22) Lahaye (J.), Marco (J.). - L'alimentation du bétail. I938, p Igo.

(23) LAPLAUd, DE LA FREGONNière, DUfFaU. - De la valeur des résultats fournis par le contrôle laitier suivant que l'on fait varier la périodicité du contrôle. Compte rendu de l'Académie d'Agriculture de France, mai I 924 .

(24) LaplaUd, DE la FRegonitìe, DUfFaU. - De la valeur des méthodes de contrôle laitier. Revue de Zootechnie, I924, vol. 3, p. 234-247.

(25) Lauprecht (E.). - Beitrag zur Frage der bei der 28 tägigen Milchkontrolle auftretenden Schwankungen der Jahresabschlüsse. Ztschr. Tierz. und Züchtungsbiol, I950, vol. $58, \mathrm{n}^{\circ}$ +, p. 5II-5I8. 
(26) M'Candlish (A. C.), M'Vicar (A.). - Are milk record association accurate? Scot. J. A gric. I925, vol. 8, $\mathrm{n}^{\circ}$ 2, p. $20 \mathrm{I}-205$.

(27) McCarthy (M. D.), Boyle (C.). - The accuracy of monthly, six weekly and two monthly weighings in estimating milk yields. $J$. Dept. A gric. Eire, I946, vol. 43, p. 5-23.

(28)* MCDOWELL (J. C.). - Testing cows for production every other month. Circ. U. S. Dep. Agric., I927, I.

(29)* Petersen (W. E.). - Accuracy of one day and two days tests. Jersey Bull., I925, vol. 44, p. $2044^{-2} 05^{8}$.

(30) Porzio (G.). - Studio comparativo sul valore dei diversi metodi di controllo della produzione lattea. Italia agricola, I933, $\mathrm{n}^{0} 4, \mathrm{p} .3-\mathrm{I} 7$.

(3I) Porzio (G.). - Il controllo del latte secondo un nuovo metodo. Italia A gricola, I953, no 6, p. 42I-427.

$(32)^{*}$ SiEdEl. - Wie oft muss man das Probemelken vornehmen? MilchZtg, I89I, vol. 20, p. II63. Bremen.

(33)* WENDT (E.). - Ueber die erforderliche Häufigkeit und den zweckmässigsten Tag des Probemelkens. Diss. Leipzig, I9I3.

(34)* Wiedersheim. - Der Wert des Probemelkens. Würt. Wochenbl. $t$. Landw., I880, vol. 3, p. 325-327, Stuttgart.

(35) YAPP (W. W.). - A study of the relative reliability of official tests for dairy cows. Bull. Ill. A gric. Exp. Sta, IgIg, no 2 I5.

$(36)^{*}$ ZorN (W.), GruHn (R.). - Milchmenge und Milchfettleistungen. Forschungsdienst., I943, vol. I6, p. 20-2I.

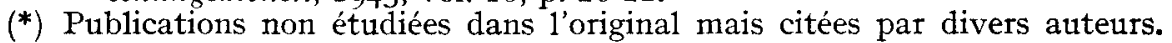
Ashton, Döring, ERB, MarcQ et Lahaye etc. 